\title{
Optimal design of an ionic liquid (IL)-based aromatic extractive distillation process involving energy and economic evaluation
}

\author{
Yang Lei ${ }^{\mathrm{a}, \mathrm{b}}$, *, Yuhang Zhou ${ }^{\mathrm{a}}$, Zhiqiang Weic, Yuqiu Chen ${ }^{\mathrm{d}, ~ *}$, Fen Guo ${ }^{\mathrm{a}}$, Wei Yan ${ }^{\mathrm{b}}$ \\ a School of Chemistry and Chemical Engineering, Hubei Key Laboratory of Coal Conversion and New Carbon \\ Materials, Wuhan University of Science and Technology, Wuhan 430081, Hubei, China \\ ${ }^{\mathrm{b}}$ Center for Energy Resources Engineering, Department of Chemistry, Technical University of Denmark, Lyngby \\ 2800, Denmark \\ c SINOPEC Refining Department, Beijing 100728, China \\ d Department of Chemical and Biochemical Engineering, Technical University of Denmark, Lyngby 2800, Denmark
}

\footnotetext{
* Corresponding author.

E-mail address: leiyang@wust.edu.cn/yanglei@kemi.dtu.dk (Y. Lei); yuqch@kt.dtu.dk (Y. Chen).
} 


\section{UNIFAC model}

\section{Model description}

UNIFAC is a functional-group based semi-empirical method for calculation of the activity coefficients. As given in Eq. A1, the activity coefficient for each component in the system consists of a combinatorial contribution $\gamma_{i}^{C}$ and a residual contribution $\gamma_{i}^{R}$.

$\ln \gamma_{i}=\ln \gamma_{i}^{C}+\ln \gamma_{i}^{R}$

The combinatorial contribution of the activity is related to the entropic effects and takes into account the size and shape of molecules, which can be described via the van der Waals volume and surface area parameters $\left(R_{k}, Q_{k}\right)$ of each functional group. The residual part of the activity is due to energetic interactions between groups present in the system, and describing the enthalpic contribution and expressed as the binary interaction parameters $\left(a_{n m}, a_{m n}\right) . \gamma_{i}{ }^{\mathrm{C}}$ and $\gamma_{i}^{R}$ are, respectively, calculated by Eqs. A2-A6 and Eqs. A7-A12.

$\ln \gamma_{i}^{C}=1-V_{i}+\ln V_{i}-5 q_{i}\left(1-\frac{V_{i}}{F_{i}}+\ln \frac{V_{i}}{F_{i}}\right)$

$V_{i}=\frac{r_{i}}{\sum_{j} r_{j} x_{j}}$

$F_{i}=\frac{q_{i}}{\sum_{j} q_{j} x_{j}}$

$r_{i}=\sum_{k} v_{k}^{(i)} R_{k}$

$q_{i}=\sum_{k} v_{k}^{(i)} Q_{k}$

where $F_{i}$ and $V_{i}$ are auxiliary properties for component $i$; relative molecular surface areas $q_{i}$ and molecular van der Waals volumes $r_{i}$ are calculated from the summation of relative group area $Q_{k}$ and relative group volume parameters $R_{k}$, respectively. $x_{j}$ is the molar composition of component $j$ in the mixture and $v_{k}^{(i)}$ is the number of groups of type $k$ in molecule $i$.

$\ln \gamma_{i}^{R}=\sum_{k} v_{k}^{(i)}\left(\ln _{\tau k}-\ln _{\tau k}^{(i)}\right)$

$\ln \Gamma_{k}=Q_{k}\left(1-\ln \left(\sum_{m} \theta_{m} \psi_{m k}\right)-\sum_{m} \frac{\theta_{m} \psi_{k m}}{\sum_{n} \theta_{n} \psi_{n m}}\right.$

$\theta_{m}=\frac{Q_{m} X_{m}}{\sum_{n} Q_{n} X_{n}}$

$X_{m}=\frac{\sum_{i} v_{m}{ }^{(i)} x_{i}}{\sum_{i} \sum_{k} v_{k}^{(i)} x_{i}}$

$\psi_{n m}=\exp \left[-\left(a_{n m} / T\right)\right]$ 
$\Psi_{m n}=\exp \left[-\left(a_{m n} / T\right)\right]$

where $\Gamma_{k}, \Gamma_{k}{ }^{(i)}$ are the residual activity coefficient of group $k$ and the residual activity coefficient of group $k$ in pure component $i$, respectively. The indices $n$ and $m$ refer to the main groups and $\Theta_{m}, X_{m}$, respectively, denote the surface area fractions and the group mole fractions. $\psi_{n m}$ and $\psi_{m n}$ are the group interaction parameters which can be calculated through Eqs. A11 and A12 from binary group interaction parameters $a_{n m}$ and $a_{m n}\left(a_{n m} \neq a_{m n}\right)$ between the functional groups $n$ and $m$. 